\title{
Obesity suppresses tumor attributable PSA, affecting risk categorization
}

\author{
Ken Chow', Stefano Mangiola1,2, Jaideep Vazirani', Justin S Peters', Anthony J Costello',3, Christopher M Hovens ${ }^{1,3}$ \\ and Niall M Corcoran $1,3,4$
}

'Departments of Urology and Surgery, Royal Melbourne Hospital and The University of Melbourne, Parkville, Victoria, Australia 2Division of Bioinformatics, Walter and Eliza Hall Institute, Parkville, Victoria, Australia

${ }^{3}$ Australian Prostate Cancer Research Centre Epworth, Richmond, Victoria, Australia

${ }^{4}$ Department of Urology, Frankston Hospital, Frankston, Victoria, Australia

Correspondence should be addressed to N M Corcoran: niallmcorcoran@gmail.com

\begin{abstract}
Obesity is linked with more aggressive prostate cancer and higher rates of disease recurrence post treatment. It is unclear if this is due to specific tumor-promoting effects of obesity or diagnostic bias. Patients undergoing prostatectomy were categorized according to their body mass index (BMI). Expected prostate-specific antigen (PSA) levels were calculated for each patient based on tumor characteristics. The effect of obesity on the accuracy of pre-treatment risk categorization was determined, and mediation analysis was used to identify the contribution of biologic vs non-biologic mechanisms to the observed increased risk of biochemical recurrence. Residual tumor-promoting effects were estimated in a survival model controlling for diagnostic error. The following results were obtained. The analysis included 1587 patients. Despite similar rates of adverse pathological features at prostatectomy, biochemical recurrence rates were significantly higher in very obese patients, which persisted after adjustment for stage, grade and PSA. Tumor volume however correlated significantly with $\mathrm{BMI}(P=0.004)$, and the difference in predicted and observed 'tumor-attributable' PSA (Delta-PSA) in very obese patients was greater than three times higher than that of healthy patients $(P=0.0067)$. Regression analysis indicated that the effect of $\mathrm{BMI}$ on tumor volume was fully mediated indirectly by its effect on PSA. Inclusion of this diagnostic error as a covariate in the survival analysis attenuated the effect of $\mathrm{BMI}$ on recurrence. In conclusion, being very obese suppresses tumor-associated PSA resulting in a diagnostic bias that is responsible for errors in risk classification, and potentially contributes to a delay in initial presentation.
\end{abstract}

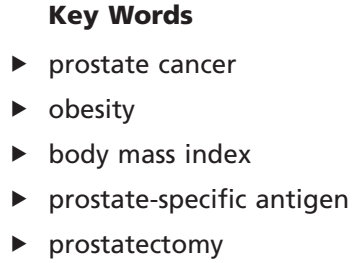

Endocrine-Related Cancer (2018) 25, 561-568

\section{Introduction}

Having a higher body mass index (BMI) has been associated with a number of solid organ tumors, including prostate cancer (Khandekar et al. 2011). Evidence accumulated over the last two decades consistently links obesity with more aggressive disease, poorer post-treatment outcomes and an increased risk of prostate cancer-specific mortality
(Buschemeyer \& Freedland 2007). The reasons for these findings however are unclear, with both diagnostic bias and obesity-related biological mechanisms implicated.

Diagnostic bias proposes that obesity increases the difficulty in making an accurate diagnosis of clinically significant prostate cancer, meaning tumors are identified 
at a later stage and/or their true risk of progression is underestimated at the time of diagnosis. The vast majority of men with prostate cancer are diagnosed based on findings of an elevated prostate-specific antigen (PSA) level and/or abnormal digital rectal examination, and there is clear evidence that obesity can affect the performance of both of these tests. For instance serum PSA levels are reported to be consistently lower in obese patients compared to healthy weight men, possibly due to hemodilution from higher plasma volume levels (Banez et al. 2007) or lower levels of circulating androgens (Klaassen et al. 2017), which may bias against early diagnosis as well as accurate risk stratification. Similarly, it is reported that a digital rectal examination, which is used both as a screening test as well as for clinical staging, is harder to perform adequately as BMI increases (Marshall 2008, Chu et al. 2011). In addition, obese patients tend to have larger prostate volumes, which may exacerbate the sampling error inherent to a standard 10- or 12-core biopsy template (Freedland et al. 2006).

The alternative, although not mutually exclusive explanation, is that the systemic molecular changes associated with the obese state contribute to an aggressive prostate cancer phenotype. Certainly adipose tissue from obese patients express a number of different growth factors and inflammatory cytokines that can promote prostate cancer growth and invasion, whereas obesityrelated changes in lipid metabolism and/or fatty acid speciation may be an important source of energy and intermediate metabolites in neoplastic prostate epithelium (Taylor et al. 2015). In addition, adipose tissue can affect circulating levels of sex steroids, which may be important in regulating tumor cell growth and survival.

In this study, we aimed to assess the relative importance of these two mechanisms on driving poorer outcomes in obese patients.

\section{Materials and methods}

\section{Patient selection}

Consecutive patients undergoing prostatectomy for clinically localized prostate cancer by participating surgeons from January 2004 to October 2012 at the Epworth Hospital Richmond were identified from a prospectively recorded dedicated database. All patients were consented prior to their procedure. Patients were excluded if key variables (BMI, tumor volume, pre-operative PSA) were not recorded and were unobtainable on retrospective review. In addition, patients treated with neo-adjuvant androgen deprivation therapy (ADT) or adjuvant ADT in the absence of recurrence were also excluded. From a total cohort of 1711 patients, 124 were excluded (neo-adjuvant ADT, $n=25$; immediate postoperative ADT, $n=18$; missing data, $n=81$ ) leaving a final study population of 1587 . Patients were categorized by their BMI, measured as $\mathrm{kg} / \mathrm{m}^{2}$, into the following categories: healthy $(\mathrm{BMI}<25)$, overweight (BMI 25 to $<30$ ), obese (BMI 30 to $<35$ ) and very obese (BMI $\geq 35)$. Biochemical recurrence was defined as a postoperative PSA of $>0.2 \mathrm{ng} / \mathrm{mL}$ or a rising PSA below this level that lead to the institution of salvage therapy. The collection and use of this data had institutional review board approval from Epworth HealthCare.

\section{Calculation of total body PSA}

To assess the potential impact of hemodilution on PSA measurements, plasma volume was estimated from height and weight measurements as previously described (Retzlaff et al. 1969), and total body PSA was calculated by multiplying PSA concentration $\times$ plasma volume.

\section{Calculation of expected tumor-related PSA}

Total tumor volume was calculated by digital planimetry as previously described (Hong et al. 2011), and for this study, no shrinkage factor was applied. For multifocal tumors, the volumes of individual tumor foci were summated to give a total tumor volume for each patient. Expected serum PSA based on the volume and grade of tumor identified at prostatectomy was calculated by multiplying the total tumor volume by the Gleason score and stage-specific PSA per tumor volume as determined previously (Stuchbery et al. 2016).

\section{Statistical analysis}

Continuous variables are expressed as medians with interquartile ranges, with differences between groups determined by Student's t-test or the Mann-Whitney $U$ test (or the Kruskal-Wallis test for multiple groups) for normally or non-normally distributed data as appropriate. Categorical variables are expressed as proportions, with intergroup differences examined using the chi-square test. To examine the differences in biochemical recurrencefree survival between groups, Kaplan-Meier curves were generated and compared using the log-rank test. Patients without recurrence were censored on the date of the last measured PSA. Cox regression was used to determine the relative importance of clinical and pathological variables 
on disease outcome. Covariates in the baseline model included tumor stage, grade, margin status and PSA. All variables entered were categorical, with the exception of pre-operative PSA, BMI and Delta-PSA (see below), which were entered as continuous variables. The effect of adding specific variables on model performance was assessed using the Omnibus Tests of Model Coefficients. To test for causation, a mediation analysis was performed using BMI as the independent variable, tumor volume as the dependent variable and Delta-PSA (the difference between expected and observed PSA based on measured tumor volume, grade and stage) as the mediator. The direct and indirect effects were measured by regression using the PROCESS macro, with confidence intervals for the indirect effect determined by bootstrapping (Hayes 2017). When Delta-PSA was included as a covariate, it was considered as measured with error, and regression coefficients corrected using the simulation-extrapolation method (Kuchenhoff et al. 2006). All analyses were performed using SPSS (IBM). All tests were two-sided with significance assumed at $P<0.05$, and where appropriate, significance at a Bonferroni adjusted $P$ value is also indicated. Based on the distribution of differences in expected and observed PSA measurements in the healthy weight group, and given the numbers of patients in each BMI category, there was $>85 \%$ power to detect a 2 -fold change with a 2 -sided alpha of $5 \%$ (Chow et al. 2008).

\section{Results}

The final study population was 1587 patients, the clinical and pathological characteristics of whom are summarized in Table 1. The average patient was overweight, had PSA detected disease and Gleason sum 7 cancer on biopsy. Follow-up from surgery was up to 12 years, with a median of 32 months during which time $23.3 \%$ recurred.

Patient characteristics categorized by BMI group are shown in Table 2. As BMI increased, patients were diagnosed at a progressively younger age, although the pre-treatment PSA as well as total PSA were equivalent across the groups. In general, patients with a healthy BMI were more likely to have clinically palpable tumors, with the exception of the very obese group who had significantly higher rates of clinical T2/T3 disease $(P=0.034)$. Similarly, patients in the very obese group had higher rates of Gleason sum $>4+3$ disease on biopsy, with the overweight and obese groups having an equivalent distribution of biopsy grades to their normal weight counterparts. At prostatectomy, all patient groups were found to have similar rates of organ-confined disease, extraprostatic extension, seminal vesicle invasion and positive pathological margins, as well as an equivalent distribution of tumor grade. Total tumor volume, however, increased significantly with BMI ( $b=0.082$, s.E. $=0.028, P=0.004)$, with the average tumor in very obese patients found to be $>50 \%$ larger than those found in patients with a BMI within the normal range (Mean cc (s.D.) of 5.09 (5.95) vs 3.27 (3.67)).

Despite broadly similar pathological characteristics at prostatectomy (with the exception of tumor volume), a higher proportion of patients in the very obese group recurred, with a significantly lower biochemical recurrencefree survival compared to the other BMI categories (Fig. 1, $P=0.0003$ ). On an initial multivariable Cox regression, we found that increasing BMI, tumor stage, Gleason sum, margin status and pre-operatively measured PSA were all independently associated with biochemical recurrence (Table 3).

Given that obesity itself has been suggested to cause difficulty with the process of prostate cancer diagnosis, we therefore considered the possibility that being very obese affected pre-treatment risk categorization independent of any biological effect on tumor progression. Pre-treatment risk categorization is commonly performed using the

Table 1 Clinical and pathological characteristics of the study cohort.

\begin{tabular}{llc}
\hline BMI $\left(\mathrm{kg} / \mathrm{m}^{2}\right)$ & Median & 27.1 \\
Clinical stage & IQR & $24.9-29.2$ \\
& T1 & $1077(67.9 \%)$ \\
& T2 & $472(29.7 \%)$ \\
PSA (ng/dL) & T3 & $38(2.4 \%)$ \\
& Median & 6.4 \\
Biopsy Gleason Sum & IQR & $4.9-8.9$ \\
& $\leq 6$ & $556(35 \%)$ \\
Prostatectomy Gleason Sum & 7 & $857(54 \%)$ \\
& $\geq 8$ & $174(11 \%)$ \\
pT & $\leq 6$ & $224(14.1 \%)$ \\
& 7 & $1216(76.6 \%)$ \\
SVI & $\geq 8$ & $147(9.3 \%)$ \\
& 2 & $1113(70.1 \%)$ \\
EPE & 3 & $474(29.9 \%)$ \\
& No & $1491(94 \%)$ \\
Pathological margin & Yes & $96(6 \%)$ \\
& No & $1120(70.6 \%)$ \\
Tumor volume (cc) & Yes & $467(29.4 \%)$ \\
& Negative & $1177(74.2 \%)$ \\
Recurrence & Positive & $410(25.8 \%)$ \\
& Median & 2.1 \\
Follow-up (months) & IQR & $1.0-4.2$ \\
& No & $1194(76.7 \%)$ \\
& Yes & $363(23.3 \%)$ \\
\hline
\end{tabular}

$E P E$, extraprostatic extension; IQR, Interquartile range; $\mathrm{pT}$, pathological T-stage; SVI, seminal vesicle invasion. 
Table 2 Clinical and pathological characteristics of study cohort categorized by BMI: healthy (BMI <25); overweight (BMI 25 to $<30$ ); obese (BMI 30 to $<35)$; very obese $(\mathrm{BMI} \geq 35)$

\begin{tabular}{|c|c|c|c|c|c|c|c|}
\hline BMI categories & & Healthy & Overweight & Obese & Very obese & $\begin{array}{c}\text { P-value (across } \\
\text { cohort) }\end{array}$ & $\begin{array}{l}\boldsymbol{P} \text {-value (BMI very } \\
\text { obese vs healthy) }\end{array}$ \\
\hline$n$ & & 419 & 870 & 252 & 46 & & \\
\hline \multirow[t]{2}{*}{ Age (year) } & Median & 62.6 & 62.1 & 60.6 & 60.6 & $0.005^{*}$ & 0.016 \\
\hline & IQR & $57.6-66.9$ & $57.2-66.5$ & $56.8-64.6$ & $55.4-63.9$ & & \\
\hline \multirow[t]{2}{*}{ PSA (ng/dL) } & Median & 6.5 & 6.2 & 6.2 & 6.6 & 0.55 & 0.82 \\
\hline & IQR & $4.9-9.3$ & $4.9-8.5$ & $4.9-8.9$ & $4.8-10.4$ & & \\
\hline \multirow[t]{2}{*}{ Total PSA (ng) } & Median & 20.3 & 20.5 & 21.2 & 23.3 & 0.62 & 0.52 \\
\hline & IQR & $15.3-28.7$ & $15.8-28.1$ & $16.5-28.8$ & $15.8-37.4$ & & \\
\hline \multirow[t]{3}{*}{ cT } & 1 & $267(63.7 \%)$ & $603(69.5 \%)$ & $180(71.4 \%)$ & $24(53 \%)$ & $0.002 *$ & 0.034 \\
\hline & 2 & $138(32.9 \%)$ & $252(29 \%)$ & $66(26.2 \%)$ & $16(36 \%)$ & & \\
\hline & 3 & $14(3.3 \%)$ & $13(1.5 \%)$ & $6(2.4 \%)$ & $5(11 \%)$ & & \\
\hline \multirow[t]{5}{*}{ Biopsy Gleason Sum } & $\leq 6$ & $154(36.8 \%)$ & $305(35.1 \%)$ & $82(32.7 \%)$ & $15(33 \%)$ & 0.24 & 0.028 \\
\hline & $\overline{7}$ & $226(54.1 \%)$ & $467(53.7 \%)$ & $140(55.8 \%)$ & $21(46 \%)$ & & \\
\hline & $3+4$ & $165(73 \%)$ & $348(74.5 \%)$ & $107(76.4 \%)$ & $11(52 \%)$ & 0.13 & 0.046 \\
\hline & $4+3$ & $61(27 \%)$ & $119(25.5 \%)$ & $33(23.6 \%)$ & $10(48 \%)$ & & \\
\hline & $\geq 8$ & $38(9.1 \%)$ & $97(11.2 \%)$ & $29(11.5 \%)$ & $10(21 \%)$ & & \\
\hline \multirow[t]{2}{*}{ pT } & 2 & $285(68 \%)$ & $626(72 \%)$ & $174(69 \%)$ & $27(59 \%)$ & 0.14 & 0.2 \\
\hline & 3 & $134(32 \%)$ & $243(28 \%)$ & $78(31 \%)$ & $19(41 \%)$ & & \\
\hline Prostatectomy & $\leq 6$ & $63(15 \%)$ & $124(14.3 \%)$ & $31(12.3 \%)$ & $6(13 \%)$ & 0.24 & 0.93 \\
\hline \multirow[t]{4}{*}{ Gleason Sum } & $\overline{7}$ & $313(74.7 \%)$ & $670(77 \%)$ & $198(78.6 \%)$ & $35(76 \%)$ & & \\
\hline & $3+4$ & $212(67.7 \%)$ & $483(72.1 \%)$ & $147(74.2 \%)$ & $19(54 \%)$ & 0.052 & 0.11 \\
\hline & $4+3$ & $101(32.3 \%)$ & $187(27.9 \%)$ & $51(25.8 \%)$ & $16(46 \%)$ & & \\
\hline & $\geq 8$ & $43(10.3 \%)$ & $76(8.7 \%)$ & $23(9.1 \%)$ & $5(11 \%)$ & & \\
\hline \multirow[t]{2}{*}{ Pathological Margin } & No & $312(74.5 \%)$ & $649(74.6 \%)$ & $187(74.2 \%)$ & $29(63 \%)$ & 0.52 & 0.1 \\
\hline & Yes & $107(25.5 \%)$ & $221(25.4 \%)$ & $65(25.8 \%)$ & $17(37 \%)$ & & \\
\hline \multirow[t]{2}{*}{ EPE } & No & $289(69 \%)$ & $629(72.3 \%)$ & $175(69.4 \%)$ & $27(59 \%)$ & 0.17 & 0.16 \\
\hline & Yes & $130(31 \%)$ & $241(27.7 \%)$ & $77(30.6 \%)$ & $19(41 \%)$ & & \\
\hline \multirow[t]{2}{*}{ SVI } & No & $391(93.3 \%)$ & $820(94.3 \%)$ & $236(93.7 \%)$ & $44(96 \%)$ & 0.87 & 0.76 \\
\hline & Yes & $28(6.7 \%)$ & $50(5.7 \%)$ & $16(6.3 \%)$ & $2(4 \%)$ & & \\
\hline \multirow[t]{2}{*}{ Tumor Volume (cc) } & Median & 2.1 & 2.0 & 2.3 & 2.3 & 0.034 & 0.06 \\
\hline & IQR & $1.0-4.0$ & $1.0-3.9$ & $1.2-5.2$ & $1.5-6.5$ & & \\
\hline \multirow[t]{2}{*}{ Recurrence } & No & $324(78.3 \%)$ & $659(77.2 \%)$ & $184(75.7 \%)$ & $27(59 \%)$ & 0.028 & $0.003 *$ \\
\hline & Yes & $90(21.7 \%)$ & $195(22.8 \%)$ & $59(24.3 \%)$ & $19(41 \%)$ & & \\
\hline
\end{tabular}

*Significant at a Bonferroni adjusted $P$-value of 0.0042 .

CT, clinical T-stage; EPE, extraprostatic extension; IQR, Interquartile range; pT, pathological T-stage; SVI, seminal vesicle invasion.

D’Amico system, which incorporates clinical stage, biopsy tumor grade and PSA to classify patients into groups with distinct natural histories and response to treatment (D’Amico et al. 1998). We first looked at concordance between clinical stage (as assessed by pre-treatment digital rectal examination) and rates of organ-confined or non-organ-confined disease at prostatectomy, and found no significant correlation between BMI category and disease under-staging at the time of diagnosis $(P=0.13$, Supplementary Table 1 , see section on supplementary data given at the end of this article). Analysis of the concordance of tumor grade between biopsy and prostatectomy similarly failed to demonstrate any significant correlation between BMI category and tumor under-grading $(P=0.91$, Supplementary Table 2). As obesity affects plasma volume resulting in PSA hemodilution (Banez et al. 2007), we then calculated the total PSA mass in each patient
(Banez et al. 2007) and observed that although this was marginally higher in the very obese group, there was no significant difference across the groups (Table $2, P=0.62$ ).

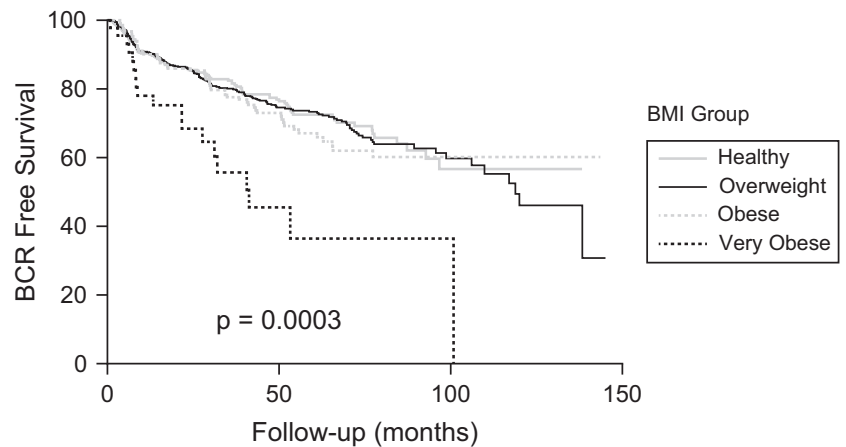

Figure 1

Kaplan-Meier curves of biochemical recurrence-free survival stratified by BMI group. 
Table 3 Multivariable, Cox regression analysis of predictors of biochemical recurrence with measured PSA as a variable.

\begin{tabular}{|c|c|c|c|}
\hline & HR & 95\% Cls & $P$-value \\
\hline BMI & 1.031 & $1.004-1.059$ & 0.026 \\
\hline \multicolumn{4}{|l|}{ T-stage } \\
\hline рT2 & 1 (Ref) & & \\
\hline pT3/4 & 2.19 & $1.73-2.77$ & $<0.001$ \\
\hline \multicolumn{4}{|l|}{ Gleason sum } \\
\hline 6 & 1 (Ref) & & \\
\hline 7 & 2.01 & $1.23-3.24$ & 0.004 \\
\hline$>8$ & 5.5 & $3.26-9.3$ & $<0.001$ \\
\hline Positive margin & 1.92 & $1.53-2.4$ & $<0.001$ \\
\hline Measured PSA & 1.023 & $1.013-1.033$ & $<0.001$ \\
\hline
\end{tabular}

HR, hazard ratio.

To determine if instead, obesity was associated with a lower than expected level of tumor PSA secretion, we calculated the concentration of serum PSA that was expected based upon the tumor volume, stage and grade (Stuchbery et al. 2016) for all individuals in the study. The observed pretreatment PSA was over-estimated in all groups; however, the difference between expected and observed PSA in very obese patients was over three times higher than that in normal weight individuals (Fig. 2) indicating that the 'real' PSA level is grossly underestimated in this cohort. This diagnostic error, the difference between expected and observed PSA, we termed Delta-PSA .

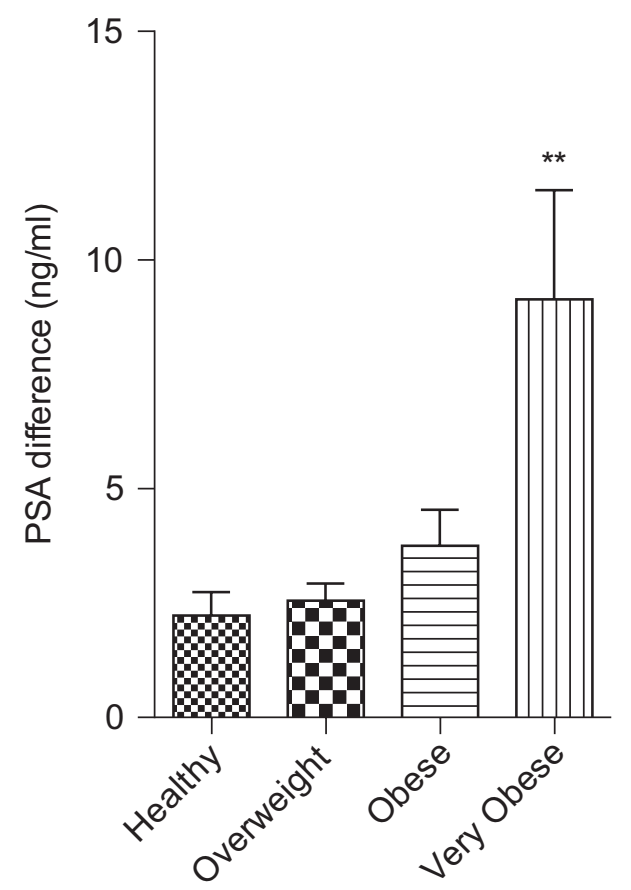

Figure 2

Difference between expected and observed serum PSA categorized by BMl group $\left({ }^{* *} P<0.01\right)$.
Table 4 Multivariable, Cox regression analysis of predictors of biochemical recurrence with Delta-PSA included as a variable.

\begin{tabular}{|c|c|c|c|}
\hline & HR & $95 \% \mathrm{Cls}$ & $P$-value \\
\hline BMI & 1.027 & $0.99-1.06$ & 0.063 \\
\hline \multicolumn{4}{|l|}{ T-stage } \\
\hline pT2 & 1 (Ref) & & \\
\hline рT3/4 & 2.24 & $1.76-2.85$ & $<0.001$ \\
\hline \multicolumn{4}{|l|}{ Gleason sum } \\
\hline 6 & 1 (Ref) & & \\
\hline 7 & 1.86 & $1.12-3.08$ & 0.016 \\
\hline$>8$ & 4.83 & $2.78-8.38$ & $<0.001$ \\
\hline Positive margin & 1.74 & $1.37-2.21$ & $<0.001$ \\
\hline Measured PSA & 1.03 & 1.02-1.04 & $<0.001$ \\
\hline Delta-PSA & 1.029 & 1.019-1.039 & 0.001 \\
\hline
\end{tabular}

$H R$, hazard ratio.

As the only adverse tumor characteristic associated with BMI was tumor volume $(b=0.082$, S.E. $=0.033$, $P=0.012$ ) and that tumor volume itself is correlated significantly with tumor grade, stage and margin status and predicts disease recurrence (Hong et al. 2011), mediation analysis was used to investigate the hypothesis that the effect of BMI on tumor volume is mediated by the diagnostic error Delta-PSA. Results indicated that BMI was a significant predictor of DeltaPSA ( $b=0.254$, s.E. $=0.08, P=0.0016)$, and that Delta-PSA was a significant predictor of tumor volume $(b=0.252$, S.E. $=0.023, P=<0.0001)$, supporting the meditational hypothesis. BMI was no longer a significant predictor of tumor volume after controlling for the mediator, Delta-PSA $(b=0.018, \quad$ s.E. $=0.022, \quad P=0.41) \quad$ consistent with full mediation. The indirect effect was tested using a bootstrap estimation approach with 5000 samples. These results determined that the indirect coefficient was significant $(b=0.064$, s.E. $=0.023,95 \%$ CIs $0.023-$ 0.11 ), indicating that the effect of BMI on tumor volume is mediated by its effect on PSA under-estimation. In very obese patients, the use of 'expected' rather than actual PSA for pre-treatment risk categorization would have resulted in a higher risk group in $23 / 46$ patients (D’Amico et al. 1998).

To determine if BMI had any residual effect on effect on the risk of recurrence after controlling for diagnostic error, we repeated the Cox regression but this time including Delta-PSA as a baseline covariate (Table 4). Addition of $\mathrm{BMI}$ to this model did not result in a significant increase in the prediction of recurrence $(P=0.066)$, and BMI was no longer an independent predictor, suggesting that any effect of BMI on tumor outcome independent of its effect on PSA is small. http://erc.endocrinology-journals.org https://doi.org/10.1530/ERC-17-0466
(C) 2018 Society for Endocrinology Published by Bioscientifica Ltd. Printed in Great Britain 


\section{Discussion}

Prostate cancer is the most commonly diagnosed male cancer in the developed world and is the third leading cause of cancer-related deaths among men (Torre et al. 2015, Siegel et al. 2018). Men in the United States have a 1 in 9 chance of being diagnosed with prostate cancer throughout their lifetime while an estimated 164,690 American men will be newly diagnosed in 2018. In 2015, prostate cancer was the second leading cause of cancer mortality in American men with 28,848 deaths (Siegel et al. 2018). With such exceptionally high prevalence of obesity and prostate cancer in the community, a possible link between the two conditions has been an area of intense investigation and is certainly supported by a number of meta-analyses that link obesity significant increases in overall prostate cancer incidence (Bergstrom et al. 2001, MacInnis \& English 2006), as well higher grade disease (Freedland et al. 2005).

Certainly, there are number of plausible biological mechanisms that potentially explain this positive association. Obesity is characterized by low-grade inflammation within adipose tissue, associated with the elaboration of numerous cytokines such as interleukin-6, monocyte chemo-attractant protein- 1 and tumor necrosis factor $\alpha$, which have all been shown to be associated with prostate development or progression (Taylor et al. 2015). Additionally, adipose tissue can produce a number of specific paracrine signaling molecules (adipokines) such as leptin and adiponectin, which are deranged in obesity and have previously been shown to affect prostate tumor cell growth (Taylor et al. 2015). Importantly, periprostatic tissue derived from overweight/obese men has been shown to promote prostate cancer cell migration and survival (Ribeiro et al. 2012, Laurent et al. 2016) and access to the periprostatic compartment (and presumably increased exposure to adipose tissue-derived tumorpromoting factors) is a more important determinant of clinical outcome than acquiring the ability to invade across tissue boundaries (Kapoor et al. 2013).

An alternative explanation for the association between obesity and poorer prostate outcomes is the suggestion that obesity may impair diagnosis, leading to later presentations with more advanced disease. For instance, it has been suggested that digital rectal examinations (DRE) are more challenging to perform on obese men and are less likely to result in an abnormal finding until the tumor is of a more aggressive nature. This is supported by a study of over 2500 men where risk for overall detection of prostate cancer with an abnormal DRE compared with normal DRE in obese men was significantly greater than that of healthy men (OR 3.43, CI 1.11-2.21, $P=0.011$; vs OR 1.57, CI 2.42-4.86, $P=<0.001$ ) (Chu et al. 2011). Studies have also demonstrated another potential reason for under-diagnosis in which obese men have larger prostates; potentially leading to lower cancer detection as regular 12-core prostate biopsy might not sample the tumor in a larger prostate. Freedland et al. demonstrated that men with BMI 30-34.9 had prostate weights of $41.4 \mathrm{~g}$ compared to $33.8 \mathrm{~g}$ in men with $\mathrm{BMI}<25$ (Freedland et al. 2006). Ochiai et al. also found that BMI had a positive correlation with prostate volume $(r=0.138, P=<0.01)$ (Ochiai et al. 2005). There may also be a diagnostic bias toward more aggressive cancers in obese men as there is evidence showing that increasing BMI is inversely proportional to PSA measurements; therefore, obese individuals are less likely to have a biopsy until the cancer is more advanced. A proposed theory for this phenomenon is that obese patients have lower measured PSA levels due to a hemodilutional effect from higher plasma volume levels (Banez et al. 2007). Under-diagnosis of prostate cancer is a real multifactorial problem for obese men, which requires further development of risk stratification methodologies.

Despite similar rates of adverse pathological features in our cohort when stratified by BMI, the very obese group had a significantly higher biochemical recurrence rate and shorter biochemical recurrence-free survival compared to other groups. The sole exception to this was total tumor volume, which correlated positively with BMI. When we looked at the association of clinical and pathological variables with the risk of biochemical recurrence in an initial multivariable model, we found that increasing BMI, clinical stage, biopsy grade and PSA were all independently associated with recurrent disease. Although we have previously shown that tumor volume at prostatectomy is independently associated with biochemical recurrence in locally advanced disease (Hong et al. 2011), the magnitude of effect (HR 1.12 per 1 cc increase in tumor volume) was insufficient to explain the significantly higher rates of recurrence demonstrated in the very obese cohort. Therefore, we postulated that being very obese in itself could potentially affect pretreatment risk categorization independent of obesityrelated biological mechanisms.

As obesity affects plasma volume resulting in PSA hemodilution (Banez et al. 2007), we first calculated the total PSA mass in each patient and observed that although this was higher in the very obese group, it was not significantly so. By utilizing our previous findings by 
Stuchbery et al., we were able to calculate the 'expected' PSA, as described in our methods, to provide an estimate of pre-treatment serum tumor attributable PSA (Stuchbery et al. 2016). We found that difference between expected and observed PSA in the comparison of very obese to healthy men was significantly greater by over threefold, demonstrating that the 'real' PSA in very obese men was significantly underestimated, independent from the previously published concept of hemodilution. Formal mediation analysis indicated that this error (Delta-PSA) fully mediated the effect the association between BMI and tumor volume, and once this error was included as a covariate in the multivariable model, BMI was no longer a predictive factor for biochemical recurrence. This suggests that obesity has an independent effect on pre-treatment categorization, and any residual effect on promoting tumor recurrence is small.

Previous studies have suggested that there is an inverse relationship between BMI and PSA. Baillargeon et al. examined this association in a population-based study of 2779 men. It concluded that serum PSA levels linearly decreased from $1.01 \mathrm{ng} / \mathrm{mL}$ in healthy weight men to $0.81 \mathrm{ng} / \mathrm{mL}$ in very obese men $(P$ trend $=<0.0001)$ (Baillargeon et al. 2005). Similarly, Bargawi et al. analyzed the data from the American nationwide Prostate Cancer screening program and discovered that the mean PSA for obese men was significantly lower than non-obese men (1.42 vs $1.78, P=<0.01)$ (Barqawi et al. 2005). Banez et al. examined 13,634 men over three main cohorts; they found that men with $\mathrm{BMI} \geq 35$ had up to $21 \%$ lower PSA concentrations than men with $\mathrm{BMI}<25$ (Banez et al. 2007). Despite studies demonstrating an association between BMI and PSA, the exact mechanism for this has not been established.

Given that testosterone is aromatized to estrogen in adipose tissue (Schulte et al. 2014) and that KLK3/PSA expression is androgen regulated, one potential explanation for the lower PSA levels in obesity is a relative reduction in circulating testosterone levels. Certainly, multiple studies have demonstrated consistently lower levels of circulating testosterone in obese individuals (Bellastella et al. 1988, Zumoff et al. 1990, Ng Tang Fui et al. 2013), and caloric restriction with subsequent reduction in adipose tissue mass can result in normalization of these levels (Schulte et al. 2014). However, a recent analysis of participants in the REDUCE study based on $\mathrm{BMI}$ indicates that the measured reduction in circulating testosterone alone in men with higher BMI only accounts for 19\% of the lower PSA observed, indicating that other obesity-related factors are important (Klaassen et al.
2017). Further research is required to determine exactly what these factors are, however, given the association between various cytokines/adipokines and prostate cancer progression as described earlier, it is possible they are promoting tumor growth independent of canonical androgen receptor signaling.

A limitation of this study is that patient selection was from a single institution in Australia, and therefore, it may not be possible to generalize these findings for different patient demographics throughout the world. The group of interest, very obese individuals, is limited by its relative small sample size. This study provides an initial insight; however, further studies should be undertaken with a larger population from multiple international institutions. In addition, we have not identified the cause of the large disparity between expected and observed PSA in very obese patients and can only speculate on potential mechanisms.

\section{Conclusion}

We have investigated the impact of BMI on expected serum PSA levels based on tumor characteristics at the time of diagnosis, and identified that in very obese individuals, there is a significant discrepancy between measured and expected levels, which may be responsible for errors in risk classification and has the potential to contribute to a delay in initial presentation.

Supplementary data

This is linked to the online version of the paper at https://doi.org/10.1530/ ERC-17-0466

\section{Declaration of interest}

The authors declare that there is no conflict of interest that could be perceived as prejudicing the impartiality of the research reported.

\section{Funding}

This research was supported by a federal grant to the Australian Prostate Cancer Research Centre from the Department of Health and Ageing. N M C is supported by a David Bickart Clinician Researcher Fellowship from the Faculty of Medicine, Dentistry and Health Sciences at the University of Melbourne.

\section{References}

Baillargeon J, Pollock BH, Kristal AR, Bradshaw P, Hernandez J, Basler J, Higgins B, Lynch S, Rozanski T, Troyer D, et al. 2005 The association of body mass index and prostate-specific antigen in a population- 
based study. Cancer 103 1092-1095. (https://doi.org/10.1002/ cncr.20856)

Banez LL, Hamilton RJ, Partin AW, Vollmer RT, Sun L, Rodriguez C, Wang Y, Terris MK, Aronson WJ, Presti JC Jr, et al. 2007 Obesityrelated plasma hemodilution and PSA concentration among men with prostate cancer. JAMA 298 2275-2280. (https://doi.org/10.1001/ jama.298.19.2275)

Barqawi AB, Golden BK, O'Donnell C, Brawer MK \& Crawford ED 2005 Observed effect of age and body mass index on total and complexed PSA: analysis from a national screening program. Urology 65 708-712. (https://doi.org/10.1016/j.urology.2004.10.074)

Bellastella A, Sinisi AA, Venditto T, Sinisi AM, Iorio S, Modafferi R, Parlato F \& Faggiano M 1988 Changes of plasma testosterone, estradiol and prolactin levels in severe adult-onset obesity. Minerva Endocrinologica 13 107-110.

Bergstrom A, Pisani P, Tenet V, Wolk A \& Adami HO 2001 Overweight as an avoidable cause of cancer in Europe. International Journal of Cancer 91 421-430. (https://doi.org/10.1002/10970215(200002)9999:9999<::AID-IJC1053>3.0.CO;2-T)

Buschemeyer WC 3rd \& Freedland SJ 2007 Obesity and prostate cancer: epidemiology and clinical implications. European Urology $\mathbf{5 2}$ 331-343. (https://doi.org/10.1016/j.eururo.2007.04.069)

Chow S-C, Shao J \& Wang H 2008 Sample Size Calculations in Clinical Research. Boca Raton, FL, USA: Taylor \& Francis.

Chu DI, De Nunzio C, Gerber L, Thomas JA 2nd, Calloway EE, Albisinni S, Senocak C, McKeever MG, Moreira DM, Tubaro A, et al. 2011 Predictive value of digital rectal examination for prostate cancer detection is modified by obesity. Prostate Cancer and Prostatic Diseases 14 346-353. (https://doi.org/10.1038/pcan.2011.31)

D'Amico AV, Whittington R, Malkowicz SB, Schultz D, Blank K, Broderick GA, Tomaszewski JE, Renshaw AA, Kaplan I, Beard CJ, et al. 1998 Biochemical outcome after radical prostatectomy, external beam radiation therapy, or interstitial radiation therapy for clinically localized prostate cancer. JAMA 280 969-974.

Freedland SJ, Terris MK, Platz EA \& Presti JC Jr 2005 Body mass index as a predictor of prostate cancer: development versus detection on biopsy. Urology 66 108-113. (https://doi.org/10.1016/j. urology.2005.01.060)

Freedland SJ, Platz EA, Presti JC Jr, Aronson WJ, Amling CL, Kane CJ \& Terris MK 2006 Obesity, serum prostate specific antigen and prostate size: implications for prostate cancer detection. Journal of Urology 175 500-504. (https://doi.org/10.1016/S0022-5347(05)00162-X)

Hayes AF 2017 Introduction to Mediation, Moderation, and Conditional Process Analysis. New York, NY, USA: Guilford Press.

Hong MK, Namdarian B, Corcoran NM, Pedersen J, Murphy DG, Peters JS, Harewood L, Sapre N, Rzetelski-West K, Costello AJ, et al. 2011 Prostate tumour volume is an independent predictor of early biochemical recurrence in a high risk radical prostatectomy subgroup. Pathology 43 138-142. (https://doi.org/10.1097/ PAT.0b013e3283420155)

Kapoor J, Namdarian B, Pedersen J, Hovens C, Moon D, Peters J, Costello AJ, Ruljancich P \& Corcoran NM 2013 Extraprostatic extension into periprostatic fat is a more important determinant of prostate cancer recurrence than an invasive phenotype. Journal of Urology 190 2061-2066. (https://doi.org/10.1016/j.juro.2013.06.050)

Khandekar MJ, Cohen P \& Spiegelman BM 2011 Molecular mechanisms of cancer development in obesity. Nature Reviews Cancer 11 886-895. (https://doi.org/10.1038/nrc3174)

Klaassen Z, Howard LE, Moreira DM, Andriole GL Jr, Terris MK \& Freedland SJ 2017 Association of obesity-related hemodilution of prostate-specific antigen, dihydrotestosterone, and testosterone. Prostate 77 466-470. (https://doi.org/10.1002/pros.23285)

Kuchenhoff H, Mwalili SM \& Lesaffre E 2006 A general method for dealing with misclassification in regression: the misclassification SIMEX. Biometrics 62 85-96. (https://doi. org/10.1111/j.1541-0420.2005.00396.x)

Laurent V, Guerard A, Mazerolles C, Le Gonidec S, Toulet A, Nieto L, Zaidi F, Majed B, Garandeau D, Socrier Y, et al. 2016 Periprostatic adipocytes act as a driving force for prostate cancer progression in obesity. Nature Communications 7 10230. (https://doi.org/10.1038/ ncomms10230)

MacInnis RJ \& English DR 2006 Body size and composition and prostate cancer risk: systematic review and meta-regression analysis. Cancer Causes Control 17 989-1003. (https://doi.org/10.1007/s10552-0060049-z)

Marshall JB 2008 How adequate is digital rectal exam for prostate cancer screening at colonoscopy? Can adequacy be improved? Digestive Diseases and Sciences 53 719-722. (https://doi.org/10.1007/s10620007-9910-5)

Ng Tang Fui M, Hoermann R, Cheung AS, Gianatti EJ, Zajac JD \& Grossmann M 2013 Obesity and age as dominant correlates of low testosterone in men irrespective of diabetes status. Andrology $\mathbf{1}$ 906-912. (https://doi.org/10.1111/j.2047-2927.2013.00124.x)

Ochiai A, Fritsche HA \& Babaian RJ 2005 Influence of anthropometric measurements, age, and prostate volume on prostate-specific antigen levels in men with a low risk of prostate cancer. Urology 66 819-823. (https://doi.org/10.1016/j.urology.2005.04.040)

Retzlaff JA, Tauxe WN, Kiely JM \& Stroebel CF 1969 Erythrocyte volume, plasma volume, and lean body mass in adult men and women. Blood 33 649-661.

Ribeiro R, Monteiro C, Cunha V, Oliveira MJ, Freitas M, Fraga A, Principe P, Lobato C, Lobo F, Morais A, et al. 2012 Human periprostatic adipose tissue promotes prostate cancer aggressiveness in vitro. Journal of Experimental and Clinical Cancer Research 3132. (https://doi.org/10.1186/1756-9966-31-32)

Schulte DM, Hahn M, Oberhauser F, Malchau G, Schubert M, Heppner C, Muller N, Gudelhofer H, Faust M, Krone W, et al. 2014 Caloric restriction increases serum testosterone concentrations in obese male subjects by two distinct mechanisms. Hormone and Metabolic Research 46 283-286. (https://doi. org/10.1055/s-0033-1358678)

Siegel RL, Miller KD \& Jemal A 2018 Cancer statistics, 2018. CA: A Cancer Journal for Clinicians 68 7-30. (https://doi.org/10.3322/ caac.21442)

Stuchbery R, Macintyre G, Cmero M, Harewood LM, Peters JS, Costello AJ, Hovens CM \& Corcoran NM 2016 Reduction in expression of the benign AR transcriptome is a hallmark of localised prostate cancer progression. Oncotarget 7 31384-31392. (https://doi. org/10.18632/oncotarget.8915)

Taylor RA, Lo J, Ascui N \& Watt MJ 2015 Linking obesogenic dysregulation to prostate cancer progression. Endocrine Connections $\mathbf{4}$ R68-R80. (https://doi.org/10.1530/EC-15-0080)

Torre LA, Bray F, Siegel RL, Ferlay J, Lortet-Tieulent J \& Jemal A 2015 Global cancer statistics, 2012. CA: A Cancer Journal for Clinicians 65 87-108. (https://doi.org/10.3322/caac.21262)

Zumoff B, Strain GW, Miller LK, Rosner W, Senie R, Seres DS \& Rosenfeld RS 1990 Plasma free and non-sex-hormone-bindingglobulin-bound testosterone are decreased in obese men in proportion to their degree of obesity. Journal of Clinical Endocrinology and Metabolism 71 929-931. (https://doi.org/10.1210/jcem-71-4-929)
Received in final form 19 March 2018

Accepted 27 March 2018
C) 2018 Society for Endocrinology Published by Bioscientifica Ltd. Printed in Great Britain 